\title{
ATRIBUTOS QUÍMICOS DE SOLOS INFLUENCIADOS PELA SUBSTITUIÇÃO DO CARBONATO POR SILICATO DE CÁLCIO(1)
}

\author{
Renato Ferreira de Souza ${ }^{(2)}$, Valdemar Faquin ${ }^{(3)}$, Ruy Carvalho(4), \\ Paulo Rogério Ferreira Torres ${ }^{(5)}$ \& Adélia Aziz Alexandre Pozza ${ }^{(6)}$
}

\begin{abstract}
RESUMO
A aplicação de silicatos pode promover incrementos na capacidade de troca de cátions, favorecer a disponibilização de ânions, especialmente $\mathrm{o}_{2} \mathrm{PO}_{4}{ }^{-}$(fosfato diácido), elevar o pH, amenizar a toxidez de $\mathrm{Al}$ e, de maneira geral, incrementar a disponibilidade de nutrientes para as plantas, apresentando, porém, uma eficiência agronômica inferior em comparação aos carbonatos. Com o objetivo de avaliar o efeito da substituição do carbonato de Ca por silicato de Ca sobre as propriedades químicas dos solos, especialmente em relação à disponibilidade de $\mathrm{P}$, foram realizados quatro experimentos em casa de vegetação, num delineamento inteiramente casualizado, com quatro repetições. Os tratamentos consistiram de cinco níveis de substituição $(0,25,50,75$ e $100 \%)$ do carbonato de Ca por silicato de Ca, mantendo uma relação estequiométrica Ca:Mg de 4:1 e o mesmo conteúdo de $\mathrm{CaO}$, suficientes para elevar $\mathrm{V}=60 \%$. Os tratamentos foram aplicados em amostras de $4 \mathbf{d m}^{3}$ de Neossolo Quartzarênico órtico, Latossolo Vermelho-Amarelo textura média, Latossolo Vermelho-Amarelo textura argilosa e Latossolo Vermelho textura muito argilosa, sendo cada solo um experimento. Foram determinados os valores dos atributos químicos dos solos: $\mathrm{pH}$ em $\mathrm{H}_{2} \mathrm{O}, \mathrm{P}, \mathrm{P}$ remanescente (P-rem), $\mathrm{K}, \mathrm{Ca}$, Mg, Si, Al, H + Al, matéria orgânica (MO), Cu, Mn, Zn e B, soma de bases (S), a CTC efetiva (t), a CTC em pH 7,0 (T), a saturação por bases (V) e a saturação por $\mathrm{Al}(\mathrm{m})$, os quais foram submetidos à analise de variância e ao ajuste de modelos de regressão simples, considerando os níveis de substituição de $\mathrm{CaCO}_{3}$ por $\mathrm{CaSiO}_{3}$. Verificou-se que a substituição de carbonato por silicato promoveu aumentos significativos nos valores de $\mathrm{Si}, \mathrm{Al}, \mathrm{H}+\mathrm{Al}$ e m e redução nos valores de $\mathrm{P}$-rem, pH, S, t e V; já os valores
\end{abstract}

\footnotetext{
(1) Recebido para publicação em junho de 2007 e aprovado em abril de 2008.

(2 Professor em Ciência do Solo. Centro Federal de Educação Tecnológica de Bambuí - CEFET. CEP 38900-000 Bambuí (MG). E-mail:souzarf.ufla@gmail.com

(3) Professor Titular do Departamento de Ciência do Solo, UFLA. E-mail: vafaquin@ufla.br

(4) Professor Titular do Departamento de Química, UFLA. E-mail: ruy@ufla.br

(5) Graduando em agronomia da UFLA. E-mail: paulorogerioftorres@gmail.com

(6) Pós-Doutoranda em Ciência do Solo. Departamento de Ciência do Solo, UFLA. E-mail: alana@ufla.br
} 
de P Mehlich-1, K, Mg, MO, T, Mn, Cu e B não foram influenciados significativamente. Houve declínio na disponibilidade de Zn somente no solo RQo. A eficácia do silicato de Ca foi inferior à de carbonato de Ca na melhoria das condições químicas do solo.

Termos de indexação: competição Si x P, adsorção de P, saturação por alumínio, disponibilidade de nutrientes.

\title{
SUMMARY: SOIL CHEMICAL PROPERTIES INFLUENCED BY THE SUBSTITUTION OF CALCIUM CARBONATE BY CALCIUM SILICATE
}

\begin{abstract}
The application of silicates to soils can result in increased soil cation exchange capacity (CEC), displace anions, especially $\mathrm{H}_{2} \mathrm{PO}_{4}^{-}$(diacid phosphate), neutralize the $\mathrm{pH}$ and $\mathrm{Al}$ toxicity and, in general, increase the nutrient availability to plants. However, calcium silicates may be less efficient than calcium carbonates. To evaluate the effect of calcium carbonate substitution by calcium silicate on the soil chemical properties, especially on phosphorus availability, four experiments were conducted in an entirely randomized design with four replications, in a greenhouse. The treatments consisted of five levels $(0,25,50$, 75 , and $100 \%)$ of calcium carbonate substitution by calcium silicate, with a 4:1 Ca: $\mathrm{Mg}$ stoichiometric and the same amount of $\mathrm{CaO}$, enough to reach a $60 \%$ base saturation. The treatments were applied to $4 \mathrm{dm}^{3}$ samples of a sandy orthic Quartzarenic Neosol (Quartzpsament), a sandy loam dystrophic Red-Yellow Latosol (Oxisol), sandy clay loam dystrophic Red-Yellow Latosol (Oxisol) and a clayey dystrophic Red Latosol (Oxisol); each soil represented one experiment. The $\mathrm{pH}$ values in $\mathrm{H}_{2} \mathrm{O}, \mathrm{P}$, phosphorus in the equilibrium solution (P-rem), $\mathrm{K}, \mathrm{Ca}, \mathrm{Mg}$, Si, $\mathrm{Al}, \mathrm{H}+\mathrm{Al}$, organic matter $(\mathrm{OM}), \mathrm{Cu}, \mathrm{Mn}, \mathrm{Zn}$ and $\mathrm{B}$, sum of bases (S), effective ( $(t)$ ant total (T) CEC, base saturation (V) and Al saturation (m) were submitted to analysis of variance and simple regression models fitted as a function of $\mathrm{CaCO}_{3}$ substitution by $\mathrm{CaSiO}_{3}$ levels. It was observed that carbonate substitution by silicate promoted significant increases in the values of $\mathrm{Si}, \mathrm{Al}, \mathrm{H}+\mathrm{Al}$ and $\mathrm{m}$ and reduction in the values of P-rem, $p H, S$, $t$ and $V$. The values of Mehlich $1 P, K, M g, O M, T, M n, C u$, and $B$ were not influenced significantly. A reduction in $Z n$ availability was verified in the dystrophic orthic Quartzarenic Neosol only. Calcium silicate was less efficient than calcium carbonate in the improvement of soil chemical conditions.
\end{abstract}

Index terms: Si x P competition, phosphorus adsorption, aluminum saturation, nutrient availability.

\section{INTRODUÇÃO}

A distribuição geográfica dos fatores químicos limitantes do desenvolvimento vegetal nos solos da América tropical foi levantada por Sanches \& Salinas (1981), que relatam que mais de $70 \%$ do total são solos intemperizados, pertencentes às classes dos Latossolos e Argissolos (Embrapa, 1999). Esses solos apresentam baixa fertilidade, baixa capacidade de troca catiônica (CTC), acidez elevada, Al tóxico, alta capacidade de reter $\mathrm{P}$, cargas variáveis dependentes de $\mathrm{pH}$ associadas, principalmente, à $\mathrm{MO}$ e predomínio de caulinita e óxidos de $\mathrm{Fe}$ e $\mathrm{Al}$ na fração argila (Mielniczuk et al., 2003).

A deficiência de $\mathrm{P}$ é a limitação nutricional mais generalizada ao desenvolvimento de pastagens em solos das regiões tropicais. O processo de adsorção de
$\mathrm{P}$ pelos óxidos, hidróxidos e oxidróxidos de $\mathrm{Fe}$ e $\mathrm{Al}$ é um dos principais fatores envolvidos na imobilização desse nutriente em solos tropicais (Lopes \& Cox, 1979), sendo a goethita considerada a principal responsável pelo fenômeno de adsorção de $\mathrm{P}$ nos solos do Brasil central (Bahia Filho et al., 1983). Essa maior capacidade de adsorção de $\mathrm{P}$ pelos solos goethíticos foi, também, constatada por Curi \& Franzmeier (1984), sendo creditada à facilidade de acesso do ânion fosfato diácido aos grupos $\mathrm{OH}^{-}$de superfície. Há, nesse caso, uma troca de ligantes: o $\mathrm{H}_{2} \mathrm{PO}_{4}{ }^{-}$ocupa o sítio das hidroxilas $\left(\mathrm{OH}^{-}\right)$previamente coordenadas ao $\mathrm{Fe}^{3+}$, formando complexos de superfícies muito mais estáveis. Reside nesse fenômeno a principal causa da fixação de P por solos intemperizados (Frossard et al., 1994). Ocorrem, também, reações de adsorção de fosfato diácido em minerais de argila que apresentam grupos $\mathrm{OH}^{-}$expostos, como a caulinita, que apresenta alta 
afinidade pelo P (Mcbride, 1994). De acordo com esse autor, em solo ácido, o ânion fosfato diácido reage rapidamente com o octaedro de $\mathrm{Al}$, pela substituição dos grupos $\mathrm{OH}^{-}$localizados na superfície do mineral, formando complexos de esfera interna. Ocorrem, também, reações de precipitação do $\mathrm{P}$ com formas iônicas de $\mathrm{Al}$ e Fe em solos ácidos e Ca em meio básico, formando compostos de baixa solubilidade (Novais \& Smyth, 1999).

O manejo a que o solo é submetido, alterando os fatores envolvidos na adsorção de $\mathrm{P}$ no solo, pode reduzir a adsorção de $\mathrm{P}$, aumentando a sua disponibilidade para as culturas. A elevação do $\mathrm{pH}$ dos solos ácidos pela aplicação de calcário apresenta vantagens como baixo custo, fácil aplicação e representa a principal fonte de $\mathrm{Ca}$ e $\mathrm{Mg}$ para as pastagens. A elevação do $\mathrm{pH}$ do solo pela calagem, aumentando a concentração e atividade dos íons $\mathrm{OH}$ em solução, promove a precipitação de $\mathrm{Fe}$ e $\mathrm{Al}$, reduzindo a precipitação de P-Fe e P-Al de baixa solubilidade. Há, também, geração de cargas negativas pela desprotonação de hidroxilas expostas nas argilas e matéria orgânica, ocorrendo repulsão entre o fosfato e a superfície adsorvente (Mcbride, 1994), ou seja, a elevação do pH a valores próximos da neutralidade dificulta a formação do complexo de superfície entre $\mathrm{H}_{2} \mathrm{PO}_{4}^{-}$e o $\mathrm{Fe}^{3+}$, permanecendo mais $\mathrm{P}$ em solução disponível às plantas.

Uma técnica alternativa para a correção da acidez do solo é a substituição total ou parcial do carbonato de $\mathrm{Ca}\left(\mathrm{CaCO}_{3}\right)$ e, ou, $\mathrm{Mg}\left(\mathrm{MgCO}_{3}\right)$ pelo silicato de $\mathrm{Ca}$ $\left(\mathrm{CaSiO}_{3}\right)$ e, ou, $\mathrm{Mg}\left(\mathrm{MgSiO}_{3}\right)$, que apresentam reações semelhantes à do calcário, que, além de elevar o $\mathrm{pH}$, podem disponibilizar o ânion silicato $\left(\mathrm{H}_{3} \mathrm{SiO}_{4}^{-}\right)$, que, por sua vez, pode concorrer com o ânion fosfato diácido pelos mesmos sítios de adsorção (Carvalho et al., 2000). A hidrólise do ânion silicato promove a liberação de hidroxilas $\left(\mathrm{OH}^{-}\right)$, neutralizando os $\mathrm{H}^{+}$e elevando o $\mathrm{pH}$ do solo. Com o aumento do $\mathrm{pH}$, ocorre a precipitação do $\mathrm{Al}^{3+}$ na forma de hidróxido de $\mathrm{Al}\left(\mathrm{Al}(\mathrm{OH})_{3}\right)$, de baixa solubilidade e, portanto, não tóxica para as plantas, já que não se mantém ativa em solução (Kondörfer \& Nolla, 2003). Após a ação alcalinizante do solo pelo $\mathrm{CaSiO}_{3}$, há a formação do $\mathrm{H}_{4} \mathrm{SiO}_{4}$, que se transforma em $\mathrm{H}_{3} \mathrm{SiO}_{4}$ - em $\mathrm{pH}$ próximo de 7, e pode ser adsorvido aos óxidos de $\mathrm{Fe}$ e $\mathrm{Al}$ da fração argila, competindo com $\mathrm{O}_{2} \mathrm{PO}_{4}{ }^{-}$pelos mesmos sítios de adsorção (Hingston et al., 1972). Essa competição, conforme Carvalho et al. (2000), ocorre quando se aplica o Si antes do calcário e do $\mathrm{P}$ das fertilizações. Na ausência do calcário, conforme se propõe neste trabalho, o $\mathrm{CaSiO}_{3}$ cumprirá primeiro o papel alcalinizante, seguido da formação de $\mathrm{H}_{3} \mathrm{PO}_{4}^{-}$, que parece saturar os sítios de adsorção de $\mathrm{H}_{2} \mathrm{PO}_{4}^{-}$, disponibilizando-o em maiores concentrações às plantas na solução do solo.

Objetivou-se avaliar o efeito da substituição parcial ou total do $\mathrm{CaCO}_{3}+\mathrm{MgCO}_{3}$ por $\mathrm{CaSiO}_{3}+\mathrm{MgSiO}_{3}$ sobre os atributos químicos, com ênfase no $\mathrm{P}$, em quatro solos bem drenados, com diferentes texturas, mineralogias e teor de matéria orgânica.

\section{MATERIAL E MÉTODOS}

Quatro experimentos foram realizados no Departamento de Ciência do Solo da Universidade Federal de Lavras, com amostras de quatro solos bem drenados, predominantes no Estado de Minas Gerais e com ampla variação nos conteúdos de argila, mineralogia e teores de matéria orgânica, constituindo cada solo um experimento específico. Para abranger ampla variação nas características físicas, químicas e mineralógicas, foram utilizados os solos classificados no Sistema Brasileiro de Classificação de Solos (Embrapa, 1999) como: Neossolo Quartzarênico órtico, fase cerrado (RQo); Latossolo Vermelho-Amarelo distrófico textura média, fase floresta tropical subperenifólia (LVAd-1); Latossolo Vermelho-Amarelo distrófico textura argilosa, fase floresta tropical subperenifólia (LVAd-2); e Latossolo Vermelho distrófico textura muito argilosa, fase floresta tropical subperenifólia (LVd).

As amostras dos solos foram coletadas na profundidade de 0-20 cm, sob vegetação natural, após retirar a vegetação e os restos orgânicos da superfície. Após a coleta, as amostras foram caracterizadas física, química e mineralogicamente (Quadro 1). A granulometria dos solos foi determinada pelo método da pipeta (Day, 1965), empregando-se $\mathrm{NaOH}$ $0,1 \mathrm{~mol} \mathrm{~L}^{-1}$ como dispersante químico e agitação rápida, sendo a fração areia $(2-0,053 \mathrm{~mm})$ separada por meio de tamisagem. O pH em água, $\mathrm{Ca}, \mathrm{Mg}, \mathrm{Al}$, $\mathrm{P}, \mathrm{K}, \mathrm{Cu}, \mathrm{Fe}, \mathrm{Mn}$ e $\mathrm{Zn}$ foi determinado conforme Embrapa (1997), sendo o Ca, Mg e Al extraídos com $\mathrm{KCl} 1 \mathrm{~mol} \mathrm{~L}^{-1}$, e P, K e os micronutrientes catiônicos pelo $\mathrm{HCl} 0,05 \mathrm{~mol} \mathrm{~L}^{-1}+\mathrm{H}_{2} \mathrm{SO}_{4} 0,0125 \mathrm{~mol} \mathrm{~L}^{-1}$ (Mehlich-1). Também foram determinados a acidez potencial $(\mathrm{H}+\mathrm{Al})$ e o carbono orgânico, conforme Raij et al. (1987); o P remanescente conforme Alvarez V. et al. (2000); e a capacidade máxima de adsorção de $\mathrm{P}$ segundo Olsen \& Watanabe (1957). Os valores de P adsorvido foram ajustados à isoterma de Langmuir para estimativa da CMAP. Na fração argila, foram quantificadas caulinita $(\mathrm{Ct})$ e gibbsita $(\mathrm{Gb})$ pela Análise Termo-Diferencial (ATD), sendo as amostras da mesma fração submetidas à difração de raios $\mathrm{X}$ (método do pó) (Klug \& Alexander, 1974). Foram determinados os óxidos de Fe livre totais $\left(\mathrm{Fe}_{\mathrm{d}}\right)$, usando o ditionito-citrato-bicarbonato de $\mathrm{Na}$ (DCB) (Mehra \& Jackson, 1960) e óxidos de Fe menos cristalinos $\left(\mathrm{Fe}_{\mathrm{o}}\right)$ extraídos segundo método de Schwertmann (1964), usando-se oxalato ácido de amônio. Em ambos os casos, a determinação do $\mathrm{Fe}$ foi realizada pela espectrofotometria de absorção atômica (Krishna Murti et al., 1966). Os óxidos foram determinados pelo ataque sulfúrico $\left(\mathrm{SiO}_{2}, \mathrm{Al}_{2} \mathrm{O}_{3}, \mathrm{Fe}_{2} \mathrm{O}_{3}, \mathrm{TiO}_{2}\right.$ e $\left.\mathrm{P}_{2} \mathrm{O}_{5}\right)$, segundo Embrapa (1997) e determinadas as relações moleculares $\mathrm{Ki}=\mathrm{SiO}_{2} / \mathrm{Al}_{2} \mathrm{O}_{3}$ e $\mathrm{Kr}=\mathrm{SiO}_{2} / \mathrm{Al}_{2} \mathrm{O}_{3}+$ $\mathrm{Fe}_{2} \mathrm{O}_{3}$.

Os experimentos foram conduzidos em casa de vegetação, utilizando-se o delineamento experimental inteiramente casualizado, com três repetições, sendo 
Quadro 1. Principais atributos químicos, físicos e mineralógicos das amostras dos solos coletadas, sob condições naturais, na camada de $0-20 \mathrm{~cm}$

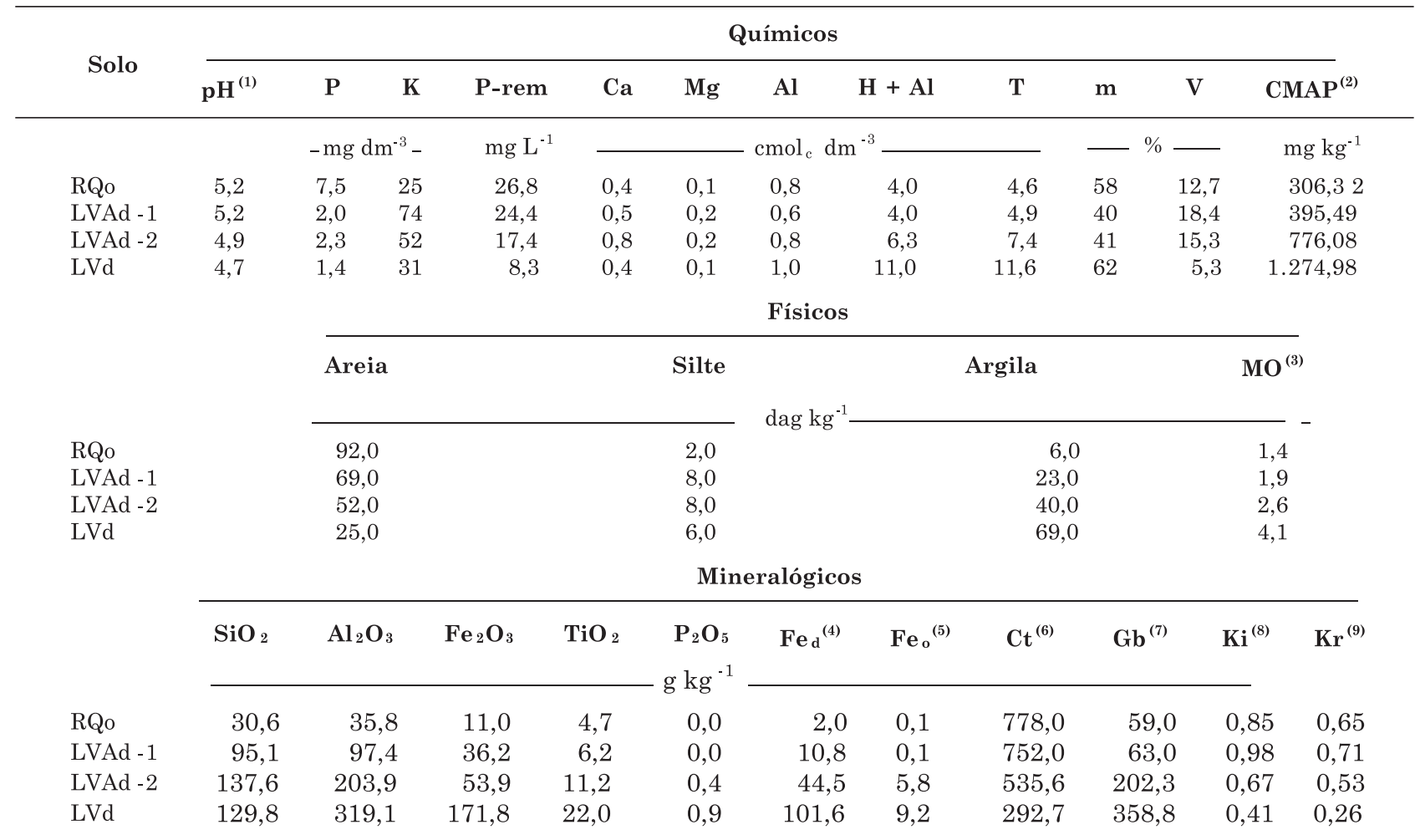

${ }^{(1)} \mathrm{pH}$ em água. ${ }^{(2)}$ Capacidade máxima de adsorção de $\mathrm{P} .{ }^{(3)} \mathrm{MO}=\% \mathrm{C}$ x 1,723. ${ }^{(4)}$ Óxidos de Fe livre totais. ${ }^{(5)}$ Óxidos de Fe menos cristalinos. ${ }^{(6)}$ Caulinita. ${ }^{(7)}$ Gibbsita. ${ }^{(8)} \mathrm{Ki}=\mathrm{SiO}_{2} / \mathrm{Al}_{2} \mathrm{O}_{3} \cdot{ }^{(9)} \mathrm{Kr}=\mathrm{SiO}_{2} / \mathrm{Al}_{2} \mathrm{O}_{3}+\mathrm{Fe}_{2} \mathrm{O}_{3}$.

cada unidade experimental constituída por um vaso com capacidade de $4 \mathrm{dm}^{3}$ de solo. Os tratamentos foram constituídos de cinco níveis $(0,25,50,75$ e $100 \%$ ) de substituição do carbonato de $\mathrm{Ca}+$ carbonato de $\mathrm{Mg}\left(\mathrm{CaCO}_{3}+\mathrm{MgCO}_{3}\right)$ por silicato de $\mathrm{Ca}+$ silicato de $\mathrm{Mg}\left(\mathrm{CaSiO}_{3}+\mathrm{MgSiO}_{3}\right)$ usando reagentes p.a., mantendo-se uma relação estequiométrica $\mathrm{Ca}: \mathrm{Mg}$ de 4:1 e o mesmo teor de $\mathrm{CaO}$ nos tratamentos, suficientes para atingir a saturação por bases em cada solo a $60 \%$. Também foi conduzido um tratamento com o solo não corrigido. Após 60 dias de incubação do solo com os corretivos e a umidade mantida próxima da capacidade de campo, subamostras foram coletadas para análise dos atributos químicos do solo: $\mathrm{pH}$ em $\mathrm{H}_{2} \mathrm{O}$, fósforo (P), P remanescente (P-rem), $\mathrm{K}, \mathrm{Ca}, \mathrm{Mg}$, $\mathrm{Si}, \mathrm{Al}, \mathrm{H}+\mathrm{Al}, \mathrm{MO}, \mathrm{Cu}, \mathrm{Mn}, \mathrm{Zn}$ e B. Foram determinados a soma de bases (S), a CTC efetiva (t), a CTC em pH 7,0 (T), a saturação por bases (V) e a saturação por $\mathrm{Al}(\mathrm{m})$. O P-rem foi determinado na solução de equilíbrio solo - solução, obtida pela agitação de $5 \mathrm{~cm}^{3}$ de TFSA com $50 \mathrm{~mL}$ de uma solução de $\mathrm{CaCl}_{2}$ $10 \mathrm{mmol} \mathrm{L}^{-1}$ contendo $60 \mathrm{mg} \mathrm{L}^{-1}$ de $\mathrm{P}$, por uma hora (Alvarez V. et al., 2000). Após centrifugado e filtrado, o P na solução de equilíbrio foi dosado pelo método da vitamina C, modificado por Braga \& Defelipo (1974); o Si solúvel em $\mathrm{CaCl}_{2}$ 0,0025 mol L-1 foi determinado segundo Raij \& Camargo (1973).
Os dados foram submetidos à análise de variância e ao estudo de regressão simples, ajustando-se modelos em função dos tratamentos aplicados com o programa SISVAR (Ferreira, 2000).

\section{RESULTADOS E DISCUSSÃO}

Embora tenha sido verificada redução significativa dos valores de P-rem, P Mehlich-1 e K, a magnitude de variação foi muito pequena (Figura 1). Em relação à disponibilidade de $\mathrm{P}$, observaram-se comportamentos semelhantes e não significativos da aplicação de corretivos, comparado ao solo não corrigido. Verificouse redução nos teores de $\mathrm{Mg}$ e Ca devido ao aumento na proporção de silicato em relação ao carbonato, indicando maiores solubilidade e efetividade do carbonato em relação ao silicato como corretivo do solo (Figura 1).

O emprego do silicato de $\mathrm{Ca}\left(\mathrm{CaSiO}_{3}\right)$ e, ou, do silicato de $\mathrm{Mg}\left(\mathrm{MgSiO}_{3}\right)$ como corretivo de solo em substituição ao $\mathrm{CaCO}_{3}$ pode ser recomendado pela possível competição do Si e $\mathrm{P}$ pelo mesmo sítio de adsorção, reduzindo fixação e maior disponibilidade do P no solo (Carvalho et al., 2000). Como o ânion silicato é quimicamente adsorvido, há, a princípio, uma 


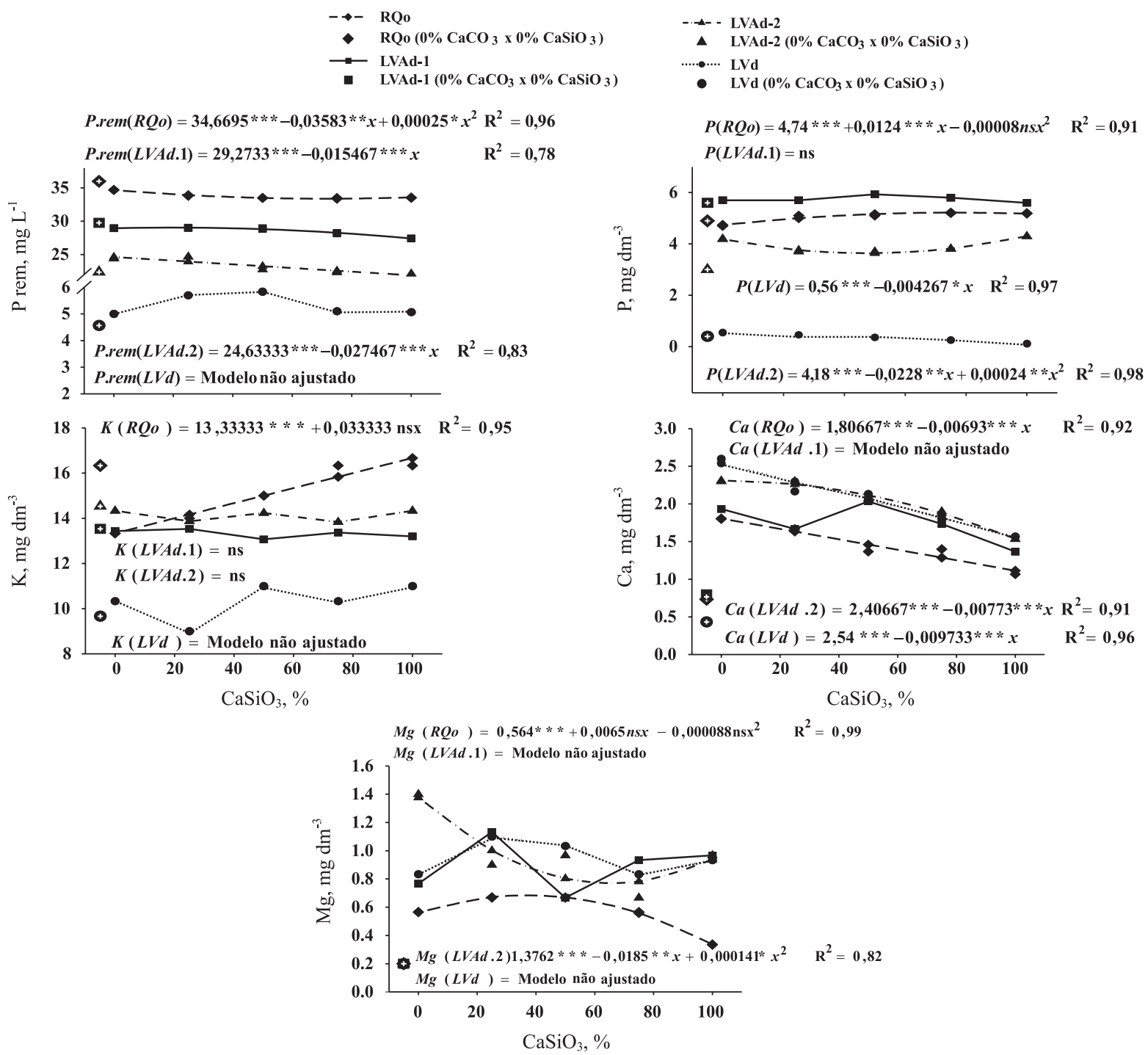

Figura 1. Influência da substituição do $\mathrm{CaCO}_{3}$ por $\mathrm{CaSiO}_{3}$ sobre os teores de P-rem, P Mehlich-1, K, Ca e Mg nos solos RQo, LVAd-1, LVAd-2 e LVd e solos sem correção. ***, **, * e ns: significativo a 0,1; 1 e 5 \%, e nãosignificativo, respectivamente.

competição entre o Si e o P pelos mesmos sítios de adsorção. Assim, com a aplicação de silicatos, além da correção da acidez, pode haver maior disponibilidade de $\mathrm{P}$ pelo efeito adicional de deslocar o $\mathrm{P}$ adsorvido para a solução (Völkweis \& Raij, 1977). Porém, esse efeito não foi observado no experimento, corroborando os dados obtidos por Carvalho et al. (2000), que, em estudo de competição Si x P, verificaram incremento de apenas $15 \%$ de $\mathrm{P}$ em solução devido ao efeito do $\mathrm{Si}$ aplicado antes da adubação fosfatada, portanto de pequena magnitude.

O efeito benéfico do Si é explicado pela substituição parcial do $\mathrm{P}$ adsorvido por Si e, conseqüentemente, pela maior disponibilidade de P no solo. Entretanto, experimentos mostraram que o Si pode não disponibilizar o $\mathrm{P}$, não reduzindo a capacidade do solo em adsorver o $\mathrm{P}$ em condições de deficiência (Carvalho et al., 2000), sugerindo um efeito insignificante do Si sobre a disponibilização do $\mathrm{P}$ no solo. Esse fato corrobora os resultados apresentados neste trabalho, no qual não ficou evidenciada uma influência da substituição do carbonato por silicato de Ca sobre o aumento na disponibilidade de $\mathrm{P}$ (Figura 1). Na solução-solo, o Si está na forma de ácido silícico, o qual não é dissociado em $\mathrm{pH}$ inferior a 9, mostrando que a interação entre Si e $\mathrm{P}$ é pouco provável. Porém, vários estudos apresentam a possibilidade de uma reação competitiva entre íons silicatos e íons fosfato pelo mesmo sítio de adsorção devido à aplicação de $\mathrm{Si}$ e ao conseqüente incremento na disponibilidade de $\mathrm{P}$ no solo (Matichenkov \& Bocharnikova, 2001). Segundo esses autores, a reação de dessorção do ânion fosfato leva ao incremento da disponibilidade de $\mathrm{P}$ em solução, criando um novo equilíbrio entre ânions silicato e fosfato no solo. À semelhança do que ocorre com o $\mathrm{P}$, o mecanismo controlador da concentração de $\mathrm{H}_{4} \mathrm{SiO}_{4}$ na fase líquida do solo parece ser governado por reações de adsorção 
dependentes de $\mathrm{pH}$ (Baldeon, 1995). Entretanto, McKeague \& Cline (1963) constataram apreciável adsorção de $\mathrm{H}_{3} \mathrm{SiO}_{4}{ }^{-}$em solos oxídicos com $\mathrm{pH}$ 4,8 em Porto Rico (EUA), o que coloca em dúvida até que ponto a adsorção de Si por solos é um fenômeno totalmente dependente de $\mathrm{pH}$. Afinal, ao contrário do $\mathrm{P}$, o $\mathrm{Si}$ permanece solúvel em uma extensa faixa de $\mathrm{pH}$ (4 a 9) que engloba os valores deste em praticamente todos os solos agrícolas.

Smyth \& Sanchez (1980), comparando a ação do $\mathrm{CaCO}_{3}$ em relação ao $\mathrm{CaSiO}_{3}$, em amostras de um Latossolo caulinítico do Cerrado brasileiro ( $\mathrm{pH} 4,8)$, mostraram redução na retenção de $\mathrm{P}$ da ordem de 18 e $24 \%$ para o $\mathrm{CaCO}_{3}$ e $\mathrm{CaSiO}_{3}$, respectivamente. Porém, Oliveira (1984), trabalhando com amostras de dois Latossolos de Minas Gerais, constatou deslocamentos (dessorção) recíprocos de Si e P, sendo verificado que $\mathrm{Si}$ foi mais facilmente deslocado pelo $\mathrm{P}$ do que o inverso, devido a uma maior energia de retenção do $\mathrm{P}$ aos sítios de adsorção, confirmando observações anteriores de Hingston et al. (1972).

Embora os solos não tenham sido comparados estatisticamente, observou-se que a magnitude dos efeitos da substituição do carbonato por silicato foi diferenciada em função do caráter oxídico dos solos. Os valores absolutos de $\mathrm{P}$-rem obedeceram à seguinte ordem crescente: LVd > LVAd-2 > LVAd-1 > RQo (Quadro 1 e Figura 1). Nesta ordem, os solos apresentam teores decrescentes de $\mathrm{MO}, \mathrm{Fe}_{\mathrm{d}}, \mathrm{Fe}_{\mathrm{o}}$, gibbsita, argila e crescentes de Ki e Kr (Quadro 1). Os valores de P-rem se correlacionam de forma significativa e negativamente com os valores de capacidade máxima de adsorção de $\mathrm{P}$ (CMAP) (Souza et al., 2006), podendo ser uma estimativa confiável da capacidade de adsorção de fosfato no solo. À medida que os solos apresentam maior teor de óxidos, aumentam a adsorção de $\mathrm{P}$ e seu conteúdo nas formas ligadas a $\mathrm{Al} \mathrm{e} \mathrm{Fe}$. Os solos que apresentaram os menores valores de $\mathrm{P}$-rem corresponderam àqueles com menores valores de $\mathrm{Ki}\left(\mathrm{SiO}_{2} / \mathrm{Al}_{2} \mathrm{O}_{3}\right)$ e $\mathrm{Kr}\left(\mathrm{SiO}_{2} /\right.$ $\mathrm{Al}_{2} \mathrm{O}_{3}+\mathrm{Fe}_{2} \mathrm{O}_{3}$ ) (Quadro 1).

Observaram-se redução nos valores de $\mathrm{pH}$, soma de bases (S), CTC efetiva (t) e saturação por bases (V) e incrementos nos valores de $\mathrm{Al}, \mathrm{H}+\mathrm{Al}$ e saturação por $\mathrm{Al}(\mathrm{m})$ com o maior nível de substituição do carbonato por silicato (Figuras 2 e 3). Não foi verificado efeito significativo da maior inclusão de silicato sobre a CTC em pH 7,0 (T) e teores de matéria orgânica (MO) dos solos estudados.

A toxidez de $\mathrm{Al}$ é um dos fatores mais limitantes para crescimento vegetal em solos ácidos. $\mathrm{O} \mathrm{Al}$ inibe o crescimento radicular e, conseqüentemente, a absorção de nutrientes. Efeitos de amenização da toxicidade de $\mathrm{Al}$ pela adição de Si têm sido reportados em muitas espécies (Cocker et al., 1998), supostamente devido à redução na concentração de $\mathrm{Al}$ tóxico. Segundo esse mesmo autor, o uso de Si para reduzir a toxidez de Al tem-se mostrado bastante efetivo. Porém, como demonstrado na figura 2 , a efetividade do silicato como corretivo do solo é inferior à do carbonato, apresentando poder de neutralização equivalente a 86 \% em relação ao carbonato de $\mathrm{Ca}$ (Vale et al., 1995)
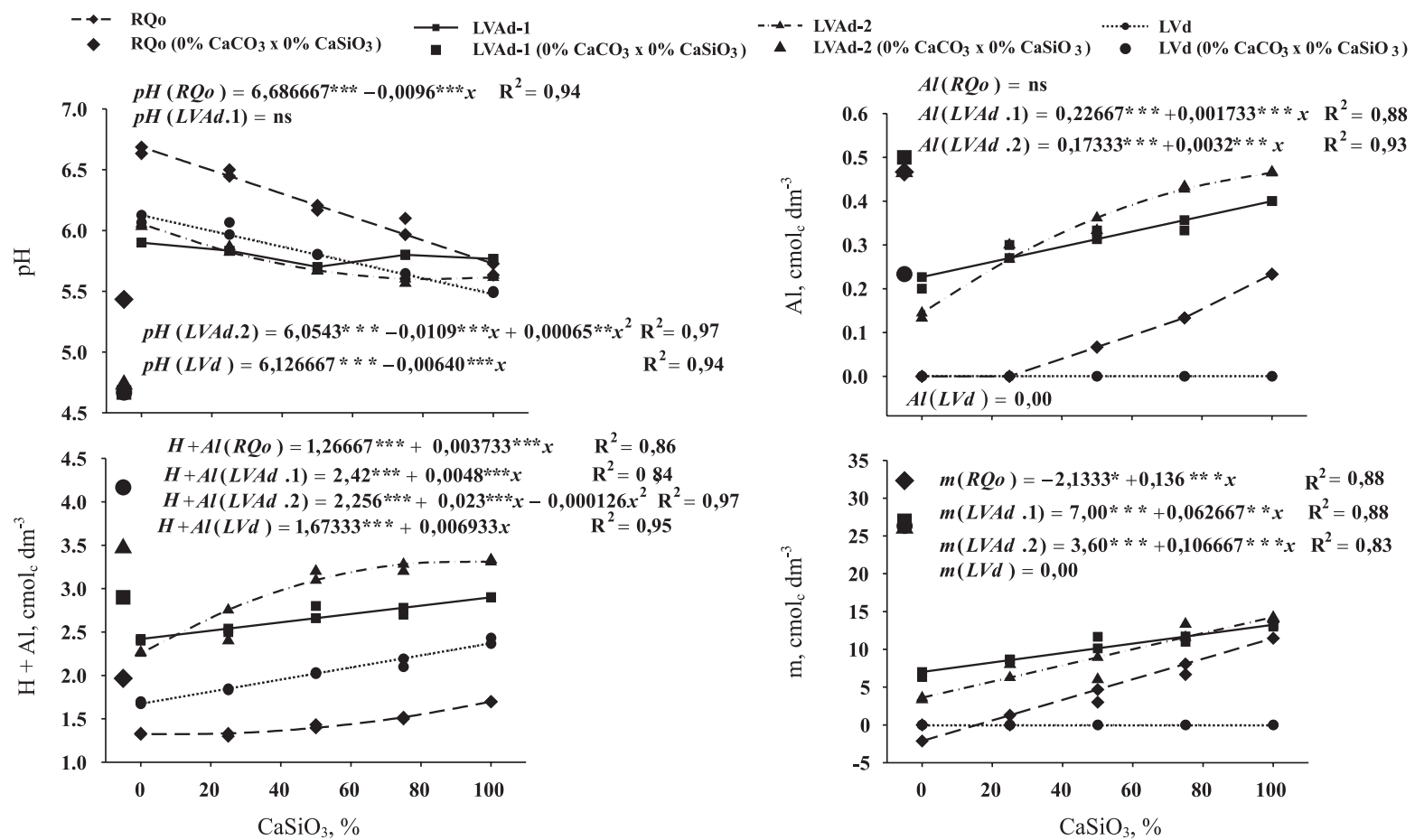

Figura 2. Influência da substituição do $\mathrm{CaCO}_{3}$ por $\mathrm{CaSiO}_{3}$ sobre os valores de pH, $\mathrm{Al}, \mathrm{H}+\mathrm{Al}$ e m nos solos RQo, LVAd-1, LVAd-2 e LVd e solos sem correção. ***, **, * e ns: significativo a 0,1; 1 e 5 \%, e nãosignificativo, respectivamente. 

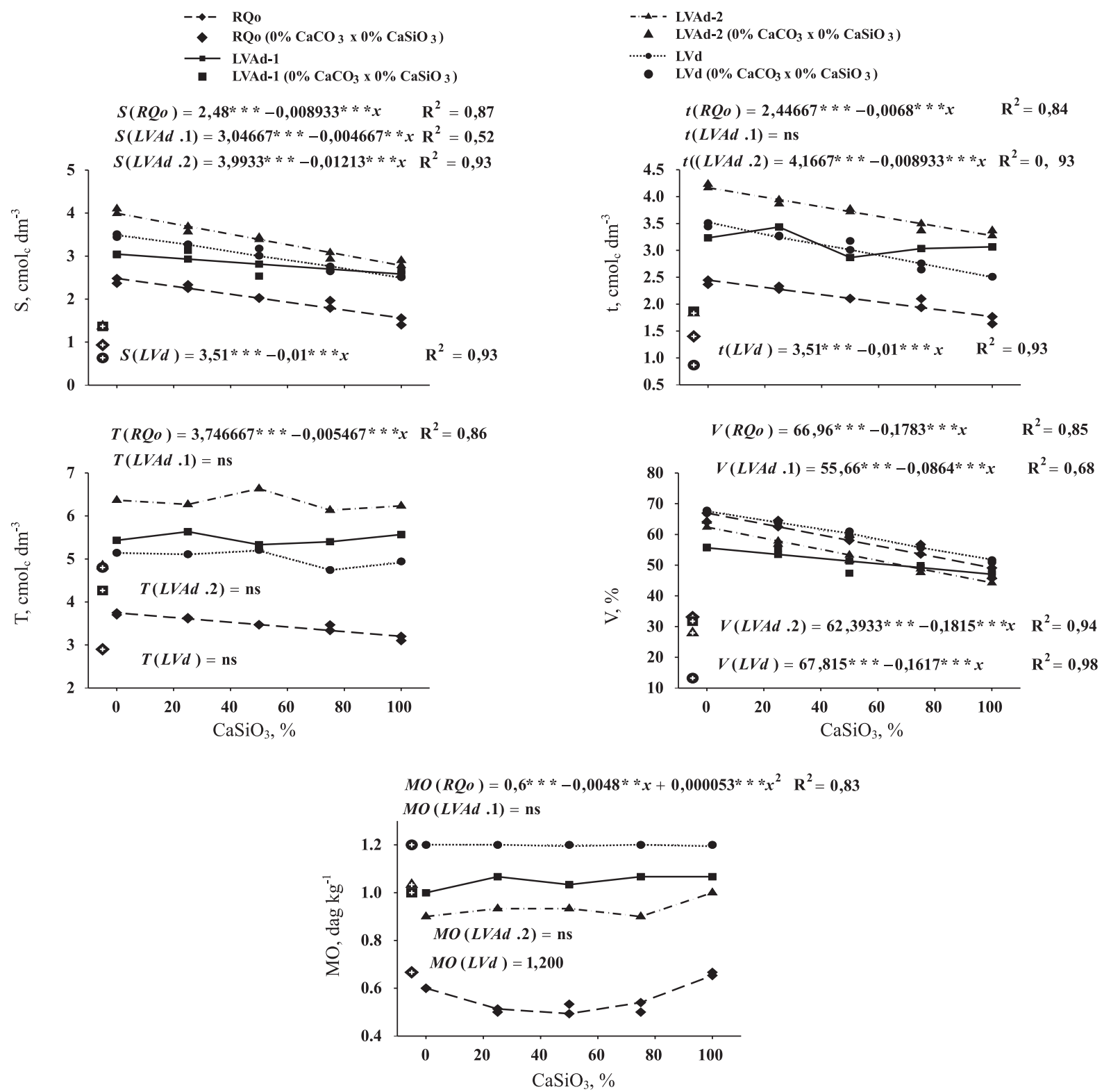

Figura 3. Influência da substituição do $\mathrm{CaCO}_{3}$ por $\mathrm{CaSiO}_{3}$ sobre os valores de $\mathrm{S}, \mathrm{t}, \mathrm{T}, \mathrm{V}$ e $\mathrm{MO}$ nos solos RQo, LVAd-1, LVAd-2 e LVd e solos sem correção. ***, **, * e ns: significativo a 0,1; 1 e 5 \% e não-significativo, respectivamente.

Os mecanismos de redução do $\mathrm{Al}$ tóxico no solo pela adição de compostos ricos em Si se dão pelo incremento do pH do solo (Carvalho et al., 2000), adsorção em hidróxidos de $\mathrm{Al}$ com conseqüente redução de sua mobilidade, formação de substâncias pouco solúveis com íons de $\mathrm{Al}$, adsorção do $\mathrm{Al}$ móvel em superfícies silicatadas e formação de compostos solúveis de Si que podem incrementar a tolerância das plantas ao $\mathrm{Al}$ tóxico (Matichenkov \& Bocharnikova, 2001). Todos esses mecanismos podem atuar simultaneamente, com alguns prevalecendo sobre os outros, dependendo das condições do solo.

Com relação aos micronutrientes estudados, verificou-se que para o $\mathrm{Mn}, \mathrm{Cu}$ e B a influência da aplicação de silicato não foi significativa. Observou- se, no solo RQo, redução nos teores de Zn com o aumento nas doses de Si. Nos outros solos, o mesmo comportamento não foi observado, verificando-se efeitos não significativos da aplicação de silicatos. A elevação dos níveis de silicato promoveu incrementos nos valores de Si no solo, principalmente no solo LVd (Figura 4).

\section{CONCLUSÕES}

1. A substituição do carbonato de Ca por silicato de Ca promoveu redução nos valores de P-rem, Ca, $\mathrm{pH}, \mathrm{S}, \mathrm{t}, \mathrm{V}$. 

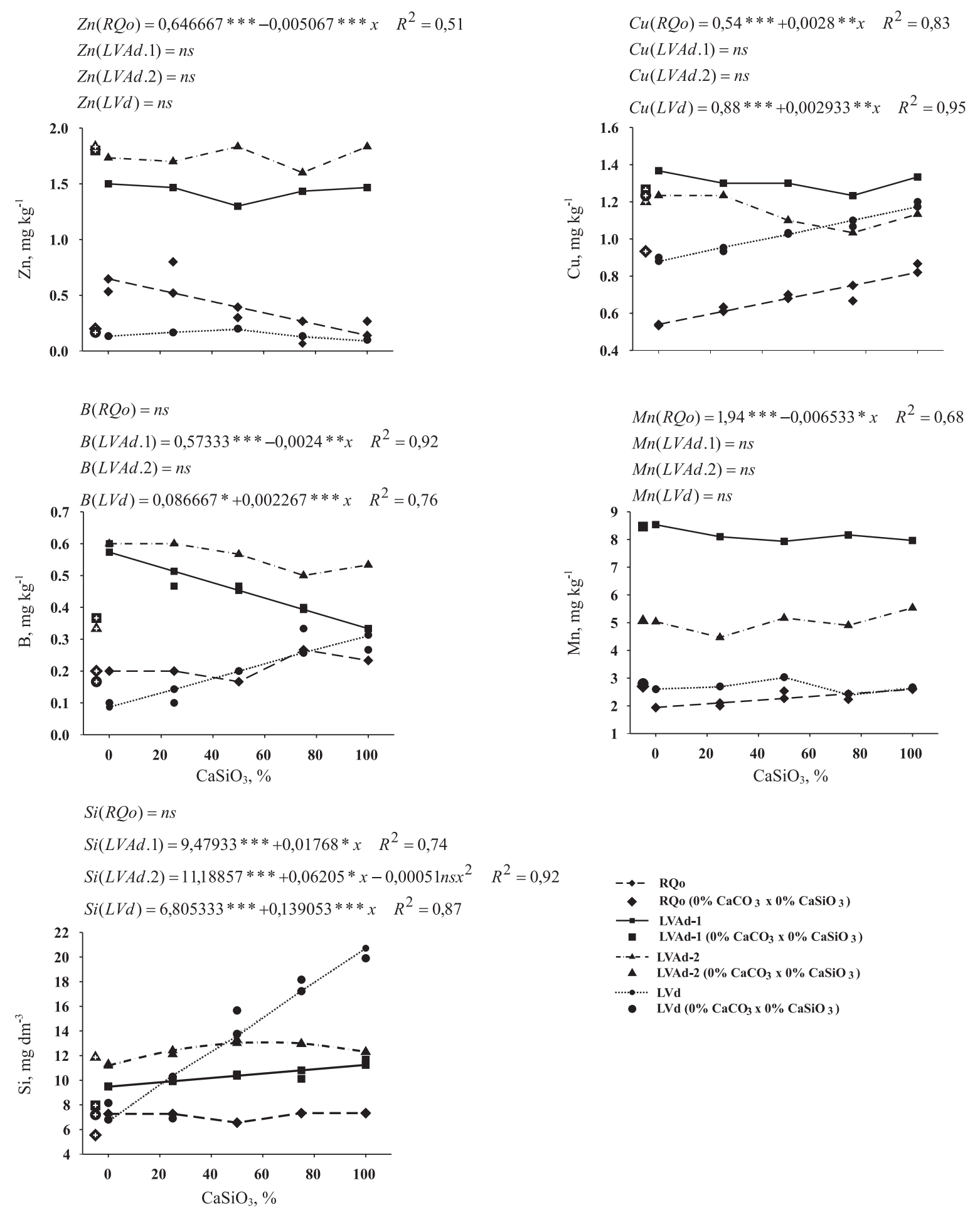

Figura 4. Influência da substituição do $\mathrm{CaCO}_{3}$ por $\mathrm{CaSiO}_{3}$ sobre os teores de $\mathrm{Zn}, \mathrm{Cu}, \mathrm{B}, \mathrm{Mn}$ e Si nos solos RQo, LVAd-1, LVAd-2 e LVd e solos sem correção. ***, **, * e ns: significativo a 0,1; 1 e 5 \%, e não-significativo, respectivamente.

2. Houve incremento nos valores de $\mathrm{Si}, \mathrm{Al}, \mathrm{H}+\mathrm{Al}$ e m devido à maior inclusão de silicato de $\mathrm{Ca}$.

3. Não houve efeito da substituição do carbonato por silicato sobre os atributos: P Mehlich-1, K, Mg, MO, T, Mn, Cu e B.
4. A maior inclusão de silicato proporcionou redução na disponibilidade de Zn no solo RQo. Nos solos LVAd-1, LVAd-2 e LVd, não foram observados efeitos significativos da maior proporção de silicato sobre os teores de Zn. 


\section{LITERATURA CITADA}

ALVAREZ V., V.H.; NOVAIS, R.F.; DIAS, L.E. \& OLIVEIRA, J.A. Determinação e uso do fósforo remanescente. B. Inf. SBCS, 25:27-32, 2000.

BAHIA FILHO, A.F.C.; BRAGA, J.M.; RESENDE, M. \& RIBEIRO, A.C. Relação entre adsorção de fósforo e componentes mineralógicos da fração argila de Latossolos do Planalto Central. R. Bras. Ci. Solo, 7:221-226, 1983.

BALDEON, J.R.M. Efeito da ação alcalinizante e da competição entre silicato e fosfato na eficiência do termofosfato magnesiano em solos ácidos. Piracicaba, Escola Superior de Agricultura Luiz de Queiroz, 1995. 88p. (Tese de Doutorado)

BRAGA, J.M. \& DEFELIPO, B.V. Determinação espectrofotométrica de fósforo em extratos de solo e material vegetal. R. Ceres, 21:73-85, 1974.

CARVAlHo, R.; FURTINE NETO, A.E.; NILTON, C. \& FERNANDES, L.A. Dessorção de fósforo por silício em solos ácidos. R. Bras. Ci. Solo, 24:69-74, 2000.

COCKER, K.M.; EVANS, D.E. \& HODSON, M.J. The amelioration of aluminum toxicity by silicon in higher plants: Solution chemistry or an in plant mechanism? Physiol. Plant., 104:608-614, 1998.

CURI, N. \& FRANZMEIER, D.P. Toposequence of Oxisols from the Central Plateau of Brazil. Soil Sci. Soc. Am. J., 48:341-346, 1984.

DAY, P.R. Particle fractionation and particle-size analysis. In: BLACK, C.A., ed. Methods of soil analysis. Madison, American Society of Agronomy, 1965. v.1. p.545-566

EMPRESA BRASILEIRA DE PESQUISA AGROPECUÁRIA EMBRAPA. Centro Nacional de Pesquisa de Solos. Sistema brasileiro de classificação de solos. Rio de Janeiro, 1999. 412p.

EMPRESA BRASILEIRA DE PESQUISA AGROPECUÁRIA EMBRAPA. Serviço Nacional de Levantamento e Conservação do Solo. Manual de métodos de análise do solo. Rio de Janeiro, 1997. 212p.

FERREIRA, D.F. Análises estatísticas por meio do Sisvar para Windows vesão 4.0. In: REUNIÃO ANUAL DA REGIÃO BRASILEIRA DA SOCIEDADE INTERNACIONAL DE BIOMETRIA, 45., São Carlos, 2000. Anais...São Carlos, Universidade de São Carlos, 2000. p.255-258.

FROSSARD, E.; BROSSARD, M.; HEDLEY, M.J. \& METHERELL, A. Reactions controlling the cycling of $\mathrm{P}$ in soils. In: TIESSEN, H., ed. P cycling in terrestrial and aquatic ecosystem: A global perspective. New York, John Wiley \& Sons, 1994. p.1-65.

HINGSTON, F.J.; POSNER, A.M. \& QUIRK, J.P. Anion adsorption by goethite and gibsite. I. The role of the proton in determining adsorption envelops. J. Soil Sci., 23:177$192,1972$.

KLUG, H.P. \& ALEXANDER, L.E. X-ray diffraction procedures for polycrystalline and amorphous materials. New York, John Wiley \& Sons, 1974. 716p.
KORNDÖRFER, G.H. \& NOLLA, A. Efeito do silício no crescimento e desenvolvimento de plantas. In: SIMPÓSIO SOBRE SÍLICIO NA AGRICULTURA, 2., Lavras, 2003. Anais. Lavras, Universidade Federal de Lavras, 2003. CDROM.

KRISHNA MURTI, G.S.R.; VOLK, V.V. \& JACKSON, M.L. Calorimetric determination of iron of mixed valency by orthophenantroline. Soil Sci. Soc. Am. Proc., 30:663-664, 1966.

LOPES, A.S. \& COX, F.R. Relação de características físicas, químicas e mineralógicas com fixação de fósforo em solos sob cerrados. R. Bras. Ci. Solo, 3:82-88, 1979.

MATICHENKOV, V.V. \& BOCHARNIKOVA, E.A. The relationship between silicon and soil physical and chemical properties. In: DATNOFF, L.E.; SNYDER, G.H. \& KORNDÖRFER, G.H. eds. Silicon in agriculture. Amsterdam, Elsevier, 2001. p.133-147. (Studies in Plant Science, 8)

McBRIDE, M.B. Environmental chemistry of soils. New York, University Press, 1994. 406p.

McKEAGUE, J.A. \& CLINE, M.G. Silica in soil solutions. Adv. Agron., 15:339-396, 1963.

MEHRA, O.P. \& JACKSON, N.L. Iron oxide removal from soils and clays by a dithionite-citrate system buffered with sodium bicarbonate. Clays Clays Miner., 3:317-327, 1960.

MIELNICZUK, J.; BAYER, C.; VEZZANI, F.M.; LOVATO, T.; FERNANDES, F.F. \& DEBARBA, L. Manejo de solo e culturas e sua relação com os estoques de carbono e nitrogênio do solo. In: CURI, N.; MARQUES, J.J.; GUILHERME, L.R.G.; LIMA, J.M.; LOPES, A.S. \& ALVAREZ V., V.H. Tópicos em ciência do solo. Viçosa, MG, Sociedade Brasileira de Ciência do Solo, 2003. v.3. p.209-248.

NOVAIS, R.F. \& SMYTH, T.J. Fósforo em solo e planta em condições tropicais. Viçosa, MG, Universidade Federal de Viçosa, 1999. 399p.

OLIVEIRA, M.G.A. Determinação, adsorção e deslocamento recíproco de silício e fósforo em Latossolo do Triângulo Mineiro. Viçosa, MG, Universidade Federal de Viçosa, 1984.68p. (Tese de Mestrado)

OLSEN, S.R. \& WATANABE, F.S. A method to determine a phosphorus adsorption maximum of soils as measured by the Langmuir Isotherm. Soil Sci. Soc. Am. Proc., 21:144$149,1957$.

RAIJ, B. van \& CAMARGO, O.A. Ślica solúvel em solos. Bragantia, 32:223-231, 1973.

RAIJ, B.van; CANTARELLA, H.; QUAGGIO, J.A.; FERREIRA, H.E.; LOPES, A.S. \& BATAGLIA, O.C. Análise química do solo para fins de fertilidade. Campinas, Fundação Cargill, 1987. 170p.

SANCHEZ, P. \& SALINAS, J.G. Low-input tecnology for managing Oxisols and Ultisols in tropical America. Adv.Agron., 34:278-406, 1981.

SCHWERTMANN, U. Differenzierung der eisenoxide des bondes durch extraktion mit ammonium-oxalat-losung. Zeitschrift Pflanzer., 105:194-202, 1964. 
SMYTH, T.J. \& SANCHEZ, P.A. Effects of lime, silicate and phosphorus application to an oxisol on phosphorus sorption and ion retention. Soil Sci. Soc. Am. J., 44:501$505,1980$.

SOUZA, R.F.; FAQUIN, V.; TORRES, P.R.F. \& BALIZA, D.P Calagem e adubação orgânica: influência na adsorção de fósforo em solos. R. Bras. Ci. Solo, 30:975-983, 2006.
VALE, F.R.; GUEDES, G.A.A. \& GUILHERME, L.R.G. Manejo da fertilidade do solo. Lavras, ESAL/FAEPE, 1995. 206p.

VÖLKWEIS, S.J. \& RAIJ, B.van. Retenção e disponibilidade de fósforo em solos. In: SIMPÓSIO SOBRE CERRADO, BASES PARA UTILIZAÇÃO AGROPECUÁRIA, 4., Brasília, 1976. Anais. São Paulo, EDUSP, 1977. p.317332. 